\title{
Hot topic: Ceramide inhibits insulin sensitivity in primary bovine adipocytes
}

\author{
J. E. Rico, ${ }^{*} \dagger$ W. A. Myers, ${ }^{*} \dagger$ D. J. Laub, ${ }^{*}$ A. N. Davis, ${ }^{*} \dagger$ Q. Zeng, ${ }^{*}$ and J. W. McFadden ${ }^{*}{ }^{1}$ \\ *Division of Animal and Nutritional Sciences, West Virginia University, Morgantown 26505 \\ †Department of Animal Science, Cornell University, Ithaca, NY 14853
}

\section{ABSTRACT}

In nonruminants, the sphingolipid ceramide inhibits insulin sensitivity by inactivating protein kinase $\mathrm{B}(\mathrm{AKT})$ within the insulin-signaling pathway. We have established that ceramide accrual develops with impaired systemic insulin action in ruminants during the transition from gestation to lactation, dietary palmitic acid supplementation, or controlled nutrient restriction. We hypothesized that ceramide promotes AKT inactivation and antagonizes insulin sensitivity in primary bovine adipocytes. Stromal-vascular cells were grown from bovine adipose tissue explants and cultured in differentiation media. To modify ceramide supply, we treated differentiated adipocytes with (1) myriocin, an inhibitor of de novo ceramide synthesis, or (2) cell-permeable C2:0-ceramide. Insulin-stimulated AKT activation (i.e., phosphorylation) and 2-deoxy-D$\left[{ }^{3} \mathrm{H}\right]$-glucose (2DOG) uptake were measured. Treatment of adipocytes with myriocin consistently decreased concentrations of ceramide, monohexosylceramide, and lactosylceramide. The insulin-stimulated ratio of phosphorylated AKT to total AKT was increased with myriocin but decreased with C2:0-ceramide. Moreover, adipocyte insulin-stimulated 2DOG uptake was decreased with $\mathrm{C} 2$ :0-ceramide and increased with myriocin. We conclude that ceramide inhibits insulinstimulated glucose uptake by downregulating AKT activation in primary bovine adipocytes.

Key words: bovine adipocyte, ceramide, insulin sensitivity

\section{Hot Topic}

The sphingolipid ceramide has received considerable attention for its ability to antagonize insulin action in obese nonruminants experiencing type 2 diabetes or nonalcoholic fatty liver disease (Holland and Summers, 2008; Pagadala et al., 2012). Specifically, nutrient excess increases de novo ceramide synthesis in adipose

Received October 12, 2017.

Accepted December 21, 2017.

${ }^{1}$ Corresponding author: jwm43@cornell.edu and liver tissues (Summers, 2006; Pagadala et al., 2012). In turn, ceramide promotes the dephosphorylation of serine-473 and inactivation of protein kinase B (AKT) to suppress glucose transporter translocation to the plasma membrane (Schubert et al., 2000). In adipocytes, impaired insulin action by ceramide promotes lipolysis (Mei et al., 2002), which is important because adipose tissue ceramide accrual and the uncontrolled lipolytic release of fatty acids are key metabolic features of heightened adiposity (Shah et al., 2008; Karpe et al., 2011). Interestingly, decreasing the activation of serine palmitoyltransferase (SPT) within the de novo ceramide synthesis pathway is a means to lower ceramide and improve localized and systemic insulin sensitivity (Holland et al., 2007).

Ceramide has emerged as an associative biomarker for impaired systemic insulin tolerance in ruminants. First, we established that the accumulation of ceramide in liver, skeletal muscle, or plasma develops with hyperlipidemia and decreased insulin sensitivity in cattle transitioning from gestation to lactation (Rico et al., 2015, 2017b). These relationships were more pronounced in cows with increased subcutaneous adiposity. Second, palmitic acid feeding increases circulating ceramide while inhibiting glucose-stimulated fatty acid disappearance (Rico et al., 2016). Third, a controlled nutrient restriction protocol to induce lipolysis increases hepatic and plasma ceramide while impairing systemic glucose tolerance (Davis et al., 2017a). Collectively, these studies repeatedly demonstrate that ceramide supply is inversely related to direct measurements of systemic insulin action; however, the ability of ceramide to mediate insulin antagonism in ruminants is uncertain. Therefore, our objective was to modulate ceramide concentrations in primary bovine adipocytes and measure insulin response.

To test our hypothesis, we utilized subcutaneous adipose tissue collected from 14 Angus or Angus-cross steers to obtain primary bovine adipocytes, using methodology developed in part by Lengi and Corl (2010). Subcutaneous adipose tissue was harvested from the flank. Stromal-vascular cells were grown from adipose tissue explants in Dulbecco's modified Eagle medium (DMEM)/F-12 growth medium containing $17.5 \mathrm{mM}$ 
glucose and $10 \%$ fetal bovine serum (FBS). Cells were harvested by trypsinization and replated, undergoing 3 or 4 passages. Seeding densities were $1 \times 10^{4}$ cells $/ \mathrm{cm}^{2}$ during the proliferation phase, and $2 \times 10^{4}$ cells $/ \mathrm{cm}^{2}$ for differentiation. Once confluent, cells were differentiated using DMEM/F-12 medium containing $5 \mathrm{mM}$ sodium acetate, $17.5 \mathrm{~m} M$ glucose, $1 \mathrm{~m} M$ octanoate, $5 \%$ FBS, $1.7 \mathrm{~m} M$ bovine insulin, $0.25 \mathrm{~m} M$ dexamethasone, $0.5 \mathrm{~m} M$ isobutylmethylxanthine, and $2 \mathrm{~m} M$ rosiglitazone. Following 8 to $10 \mathrm{~d}$ of differentiation induction, adipocytes were used in experiments and incubated in DMEM/F-12 treatment medium containing 1\% FBS and $0.6 \mathrm{n} M$ insulin. Depending on the experiment, the treatment medium contained 1 or $5 \mathrm{~m} M$ sodium acetate with 5 or $17.5 \mathrm{~m} M$ glucose, respectively. Cell differentiation was confirmed by visually observing lipid droplet formation and measuring intracellular triacylglycerol accumulation using colorimetry (kit no. K622-100; BioVision, Mountain View, CA). Cells were incubated in treatment medium in the absence or presence of myriocin $(10 \mu M$; Cayman Chemical, Ann Arbor, MI) or C2: 0-ceramide (100 $\mu M$; Cayman Chemical) for 18 or $2 \mathrm{~h}$, respectively. For future ceramide quantification, cells were washed and pelleted in ice-cold PBS. For immunoblotting purposes, cells were rinsed with $50 \mathrm{~m} M \mathrm{NaF}$ in PBS and harvested in lysis buffer $[20 \mathrm{~m} M$ Tris base, $50 \mathrm{~m} M \mathrm{NaCl}, 50 \mathrm{~m} M \mathrm{NaF}, 5 \mathrm{~m} M \mathrm{Na}_{4} \mathrm{O}_{7} \mathrm{P}_{2}, 250 \mathrm{~m} M$ sucrose, $1 \%$ (vol/vol) Triton X-100, $500 \mathrm{~m} M$ dithiothreitol, and protease inhibitor]. Pellets and cell lysates were immediately stored at $-80^{\circ} \mathrm{C}$. Insulin stimulation was performed using $200 \mathrm{nM}$ insulin for $15 \mathrm{~min}$. Assessment of 2-deoxy-D- $\left[{ }^{3} \mathrm{H}\right]$-glucose (2DOG) uptake was measured at treatment conclusion. Specifically, cells were incubated with $2 \mathrm{DOG}(0.75 \mathrm{mCi} / \mathrm{mL}$; a nonmetabolizable glucose derivative; American Radiolabeled Chemicals Inc., St. Louis, MO) and $0.1 \mathrm{~m} M$ 2-deoxyD-glucose for 5 min. Following washing with PBS, cells were harvested in $1 \%$ SDS-PBS and radioactivity was measured using a liquid scintillation counter (Beckman Coulter Inc., Brea, CA).

Ceramide, monohexosylceramide (GlcCer), and lactosylceramide (LacCer) were extracted from adipocytes using a modified Bligh and Dyer procedure and quantified using liquid chromatography (ExionLC AD; Sciex, Framingham, MA) and electrospray ionization coupled with tandem mass spectrometry (QTRAP 5500; Sciex) as previously described (Davis et al., 2017a; Phipps et al., 2017). Cell lysate protein quantification was determined using the Pierce bicinchoninic acid (BCA) protein assay kit (Thermo Scientific, Waltham, MA). For analysis of AKT phosphorylation, $50 \mu \mathrm{g}$ of protein was loaded onto a gradient gel and separated using electrophoresis. Proteins were transferred to a nitrocellulose membrane and incubated overnight with phosphorylated AKT (pAKT, Ser-473, 1:1,000; Cell Signaling Technology, Danvers, MA) or total AKT (tAKT, 1:1,000; Cell Signaling Technology) antibodies. Following washing, membranes were incubated with secondary antibody $(1: 2,000$; anti-rabbit IgG, horseradish peroxidase-linked; Cell Signaling Technology) followed by chemiluminescence detection using an Azure c600 imager (Azure Biosystems, Dublin, CA). Densitometry was performed using ImageJ (Schneider et al., 2012). Data were normalized to a loading control ( $\beta$-actin; Cell Signaling Technology; Supplemental Figure S1; https://doi.org/10.3168/jds.2017-13983). Utilizing a previous approach (Amstalden et al., 2005; McFadden and Corl, 2009), 3 independent experiments (animals) with 3 replicates per experiment were performed for triacylglycerol and ceramide quantification, whereas 2 independent experiments with 3 replicates per experiment were completed for all other measurements. Of importance, our conclusion is supported by observations utilizing cultures obtained from 4 independent experiments. Data were analyzed under a mixed model including the fixed effect of treatment and the random effect of experiment and replicate within treatment. All results are expressed as least squares means and their standard errors. Significance was declared at $P<0.05$.

Relative to undifferentiated adipocytes, triacylglycerol accumulation was $736 \%$ greater in differentiated adipocytes $(P<0.01)$ with lipid droplet formation. Differentiated adipocytes were treated with myriocin, which is a common approach to inhibit SPT and de novo ceramide synthesis (Chen et al., 1999; Holland et al., 2007). Treating primary bovine adipocytes with myriocin for $18 \mathrm{~h}$ significantly decreased ceramide, GlcCer, and LacCer concentrations (Table 1; Supplemental Figure S2; https://doi.org/10.3168/jds.2017 -13983). Although myriocin lowered all ceramides, very long chain ceramides were most responsive to SPT inhibition. For instance, myriocin decreased C24:0-ceramide concentration by $74 \%(P<0.01)$. In nonruminants, the ceramide-lowering ability of myriocin improves AKT activation, in turn improving adipocyte and wholebody insulin sensitivity (Chavez et al., 2003; Holland et al., 2007). Within our in vitro culture system, the inhibition of adipocyte de novo ceramide synthesis by myriocin developed with enhanced AKT activation, as demonstrated by an increased ratio of pAKT to tAKT (Figure 1A). To support our findings, we treated primary bovine adipocytes with $\mathrm{C} 2: 0$-ceramide, which is a cell membrane-permeable analog that inhibits insulinstimulated AKT activation in rodent models (Obanda et al., 2012; Hsieh et al., 2014). We demonstrated that treating bovine adipocytes with C2:0-ceramide for $2 \mathrm{~h}$ 
decreased the ratio of pAKT to tAKT (Figure 1B). Moreover, we confirmed that $\mathrm{C} 2$ :0-ceramide treatment decreased 2DOG uptake (Figure 1C), whereas myriocin treatment increased the transport of 2DOG into cells (Figure 1D). Notably, the ability of myriocin to improve insulin sensitivity, as measured by 2DOG uptake, was more pronounced in adipocytes cultured with higher concentrations of glucose and acetate. Although we did not investigate the effects of energy level on AKT phosphorylation or 2DOG uptake, the enhanced efficacy of myriocin to improve insulin sensitivity at a heightened energy level merits further attention, given that overnutrition impairs insulin sensitivity and promotes ceramide accrual in adipose tissue (Shah et al., 2008). Moreover, research efforts should be focused on determining whether the ability of ceramide to decrease insulin sensitivity by promoting AKT dephosphorylation of Ser-473 involves protein kinase C recruitment

Table 1. Sphingolipid concentrations in primary bovine adipocytes incubated without or with myriocin for $18 \mathrm{~h}^{1}$

\begin{tabular}{|c|c|c|c|c|}
\hline \multirow[b]{2}{*}{ Sphingolipid $^{2}$} & \multicolumn{2}{|c|}{ Treatment } & \multirow[b]{2}{*}{ SEM } & \multirow[b]{2}{*}{$P$-value } \\
\hline & Control & Myriocin & & \\
\hline \multicolumn{5}{|l|}{ Ceramide } \\
\hline $\mathrm{C} 16: 0$ & 668 & 356 & 14.3 & $<0.01$ \\
\hline C18:0 & 47.7 & 15.9 & 0.78 & $<0.01$ \\
\hline C18:1 & 41.4 & 27.9 & 1.62 & $<0.01$ \\
\hline $\mathrm{C} 20: 0$ & 20.9 & 2.96 & 1.18 & $<0.01$ \\
\hline C20:1 & 3.14 & 0.79 & 0.27 & $<0.01$ \\
\hline $\mathrm{C} 22: 0$ & 183 & 41.4 & 9.14 & $<0.01$ \\
\hline $\mathrm{C} 22: 1$ & 9.54 & 19.5 & 5.62 & 0.20 \\
\hline $\mathrm{C} 24: 0$ & 491 & 116 & 23.9 & $<0.01$ \\
\hline C24:1 & 275 & 76.5 & 4.98 & $<0.01$ \\
\hline C26:0 & 16.4 & 1.61 & 1.78 & $<0.01$ \\
\hline $\mathrm{C} 26: 1$ & 33.4 & 5.72 & 2.51 & $<0.01$ \\
\hline \multicolumn{5}{|l|}{ GlcCer } \\
\hline C16:0 & 99.6 & 37.6 & 5.39 & $<0.01$ \\
\hline C18:0 & 13.8 & 3.20 & 0.75 & $<0.01$ \\
\hline $\mathrm{C} 18: 1$ & 6.08 & 2.91 & 0.44 & $<0.01$ \\
\hline C20:0 & 10.7 & 1.37 & 0.85 & $<0.01$ \\
\hline $\mathrm{C} 22: 0$ & 72.4 & 18.0 & 5.42 & $<0.01$ \\
\hline $\mathrm{C} 24: 0$ & 108 & 43.7 & 7.23 & $<0.01$ \\
\hline C24:1 & 40.2 & 9.96 & 1.69 & $<0.01$ \\
\hline \multicolumn{5}{|l|}{ LacCer } \\
\hline C16:0 & 58.2 & 33.0 & 4.91 & $<0.01$ \\
\hline C18:0 & 6.55 & 3.79 & 0.27 & $<0.01$ \\
\hline C18:1 & 3.06 & 1.83 & 1.39 & $<0.01$ \\
\hline $\mathrm{C} 20: 0$ & 2.69 & 0.89 & 0.32 & $<0.01$ \\
\hline $\mathrm{C} 22: 0$ & 24.6 & 16.2 & 0.54 & $<0.01$ \\
\hline $\mathrm{C} 22: 1$ & 1.73 & 0.69 & 0.40 & $<0.01$ \\
\hline $\mathrm{C} 24: 0$ & 47.8 & 39.4 & 0.91 & 0.06 \\
\hline $\mathrm{C} 24: 1$ & 22.7 & 16.4 & 0.70 & $<0.01$ \\
\hline Total ceramide & 1,809 & 678 & 42.2 & $<0.01$ \\
\hline Total GlcCer & 354 & 117 & 15.8 & $<0.01$ \\
\hline Total LacCer & 169 & 115 & 4.36 & $<0.01$ \\
\hline
\end{tabular}

${ }^{1}$ Values are presented as LSM \pm SEM for each treatment.

${ }^{2}$ Sphingolipid concentrations are in nanograms of sphingolipid per milliliter of cell extract. GlcCer = monohexosylceramide (i.e., glucosyland galactosylceramide); LacCer = lactosylceramide.
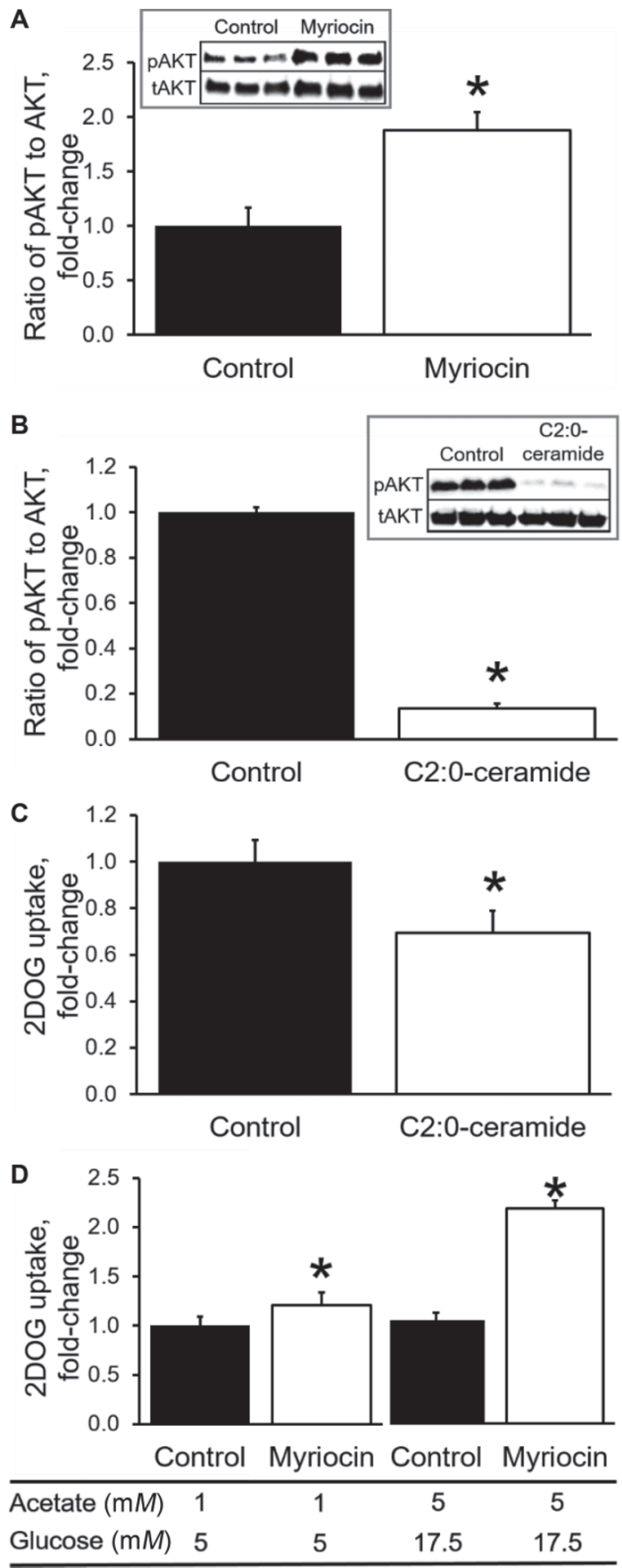

Figure 1. Ceramide inhibits protein kinase B (AKT) activation and 2-deoxy-D- $\left[{ }^{3} \mathrm{H}\right]$-glucose $(2 \mathrm{DOG})$ uptake in primary bovine adipocytes. Fold-change in the ratio of phosphorylated AKT (pAKT; Ser473) to total AKT (tAKT) in adipocytes treated with (A) myriocin, or (B) C2:0-ceramide. Fold-change in 2DOG uptake in adipocytes treated with (C) C2:0-ceramide or (D) myriocin. Cells treated with myriocin $(10 \mu M)$ for $18 \mathrm{~h}$ were cultured in medium containing $1 \mathrm{~m} M$ acetate and $5 \mathrm{mM}$ glucose unless otherwise noted within figure. Cells treated with C2:0-ceramide $(100 \mu M)$ for $2 \mathrm{~h}$ were cultured in medium containing $5 \mathrm{~m} M$ acetate and $17 \mathrm{~m} M$ glucose. For all experiments, cells were stimulated with $200 \mathrm{n} M$ insulin for 15 min immediately before harvest. Treatment effects were analyzed within energy level. Data are shown as least squares means and their standard errors. ${ }^{*} P<0.05$ : difference between treatment (myriocin or C2:0-ceramide) and control. 
and the inhibition of phosphatidylinositol 3,4,5-triphosphate binding to AKT, as observed in L6 myotubes (Powell et al., 2003).

The structural moiety and compartmental location of ceramide are determining factors for whether specific ceramides antagonize insulin sensitivity in rodents (Powell et al., 2004; Boon et al., 2013; Chavez et al., 2014). In ruminants, C24:0-ceramide in liver and plasma and C16:0-ceramide in skeletal muscle are inversely related to systemic insulin sensitivity (Rico et al., 2015, 2017b; Davis et al., 2017a). In the current study, C16:0- and C24:0-ceramide were the most abundant ceramides in primary bovine adipocytes. However, we recognize that myriocin decreased the synthesis of all ceramides, as well as glycosylated GlcCer and LacCer. Moreover, C2: 0 -ceramide is an atypical short-chain sphingolipid with low hydrophobicity that may not adequately reflect the ability of longer chain ceramides, commonly found in circulation and tissues, to modify insulin signaling. Our use of myriocin and C2:0-ceramide did not permit us to determine whether ceramides with different structural attributes inhibit AKT phosphorylation with variable efficacy. This unknown should be considered because dietary fatty acid feeding influences the ceramide profile in ruminants (Rico et al., 2016, 2017a; Davis et al., 2017b).

We conclude that ceramide inhibits AKT phosphorylation and glucose uptake in bovine adipocytes. Because considerable effort has revealed ceramide to be inversely associated with insulin sensitivity in periparturient (Rico et al., 2015, 2017b), palmitic acid-fed (Rico et al., 2016), and nutrient-restricted (Davis et al., 2017a) cattle, ceramide accrual likely contributes to insulin antagonism in ruminants. Ongoing work is aimed at confirming this possibility in vivo. Considering that some metabolic features of energy storage and adipocyte biology may differ between beef and dairy cattle (Ren et al., 2002; Bellmann et al., 2004; Hausman et al., 2014), potential variation in ceramide action between breeds merits further consideration. At stake is the potential to develop novel strategies that target de novo ceramide synthesis as a means to modify insulin sensitivity in cattle and thus influence health and production.

\section{ACKNOWLEDGMENTS}

This work was supported by the USDA Agriculture and Food Research Initiative Competitive Grant (\#WVA00907). We thank Kara Ehrenberger for her assistance with the maintenance of cell cultures and Zachary Phipps for assisting with the mass spectrometry analyses (West Virginia University).

\section{REFERENCES}

Amstalden, M., P. G. Harms, T. H. Welsh, R. D. Randel, and G. L. Williams. 2005. Effects of leptin on gonadotropin-releasing hormone release from hypothalamic-infundibular explants and gonadotropin release from adenohypophyseal primary cell cultures: Further evidence that fully nourished cattle are resistant to leptin. Anim. Reprod. Sci. 85:41-52.

Bellmann, O., J. Wegner, C. Rehfeldt, F. Teuscher, F. Schneider, J. Voigt, M. Derno, H. Sauerwein, J. Weingärtner, and K. Ender. 2004. Beef versus dairy cattle: A comparison of metabolically relevant hormones, enzymes, and metabolites. Livest. Prod. Sci. 89:41-54.

Boon, J., A. J. Hoy, R. Stark, R. D. Brown, R. C. Meex, D. C. Henstridge, S. Schenk, P. J. Meikle, J. F. Horowitz, B. A. Kingwell, C. R. Bruce, and M. J. Watt. 2013. Ceramides contained in LDL are elevated in type 2 diabetes and promote inflammation and skeletal muscle insulin resistance. Diabetes 62:401-410.

Chavez, J. A., T. A. Knotts, L. P. Wang, G. B. Li, R. T. Dobrowsky, G. L. Florant, and S. A. Summers. 2003. A role for ceramide, but not diacylglycerol, in the antagonism of insulin signal transduction by saturated fatty acids. J. Biol. Chem. 278:10297-10303.

Chavez, J. A., M. M. Siddique, S. T. Wang, J. Ching, J. A. Shayman, and S. A. Summers. 2014. Ceramides and glucosylceramides are independent antagonists of insulin signaling. J. Biol. Chem. 289:723-734.

Chen, J. K., W. S. Lane, and S. L. Schreiber. 1999. The identification of myriocin-binding proteins. Chem. Biol. 6:221-235.

Davis, A. N., J. L. Clegg, C. A. Perry, and J. W. McFadden. 2017a. Nutrient restriction increases circulating and hepatic ceramide in dairy cows displaying impaired insulin tolerance. Lipids 52:771780.

Davis, A. N., Z. C. Phipps, Q. Zeng, J. de Souza, J. E. Rico, A. L. Lock, and J. W. McFadden. 2017b. Palmitic acid feeding increases plasma ceramide concentrations in Holstein dairy cows during early lactation. J. Dairy Sci. 100(E-Suppl. 2):101. (Abstr.)

Hausman, G. J., U. Basu, S. Wei, D. B. Hausman, and M. V. Dodson. 2014. Preadipocyte and adipose tissue differentiation in meat animals: Influence of species and anatomical location. Annu. Rev. Anim. Biosci. 2:323-351.

Holland, W. L., J. T. Brozinick, L.-P. Wang, E. D. Hawkins, K. M. Sargent, Y. Liu, K. Narra, K. L. Hoehn, T. A. Knotts, A. Siesky, D. H. Nelson, S. K. Karathanasis, G. K. Fontenot, M. J. Birnbaum, and S. A. Summers. 2007. Inhibition of ceramide synthesis ameliorates glucocorticoid-, saturated-fat-, and obesity-induced insulin resistance. Cell Metab. 5:167-179.

Holland, W. L., and S. A. Summers. 2008. Sphingolipids, insulin resistance, and metabolic disease: New insights from in vivo manipulation of sphingolipid metabolism. Endocr. Rev. 29:381-402.

Hsieh, C.-T., J.-H. Chuang, W.-C. Yang, Y. Yin, and Y. Lin. 2014. Ceramide inhibits insulin-stimulated Akt phosphorylation through activation of Rheb/mTORC1/S6K signaling in skeletal muscle. Cell. Signal. 26:1400-1408.

Karpe, F., J. R. Dickmann, and K. N. Frayn. 2011. Fatty acids, obesity, and insulin resistance: Time for a reevaluation. Diabetes 60:2441-2449.

Lengi, A. J., and B. A. Corl. 2010. Factors influencing the differentiation of bovine preadipocytes in vitro. J. Anim. Sci. 88:1999-2008.

McFadden, J. W., and B. A. Corl. 2009. Activation of AMP-activated protein kinase (AMPK) inhibits fatty acid synthesis in bovine mammary epithelial cells. Biochem. Biophys. Res. Commun. 390:388-393.

Mei, J., L. S. Holst, T. R. Landström, C. Holm, D. Brindley, V. Manganiello, and E. Degerman. 2002. C2-ceramide influences the expression and insulin-mediated regulation of cyclic nucleotide phosphodiesterase 3B and lipolysis in 3T3-L1 adipocytes. Diabetes 51:631-637.

Obanda, D. N., A. Hernandez, D. Ribnicky, Y. Yu, X. H. Zhang, Z. Q. Wang, and W. T. Cefalu. 2012. Bioactives of Artemisia dracunculus L. mitigate the role of ceramides in attenuating insulin signaling in rat skeletal muscle cells. Diabetes 61:597-605. 
Pagadala, M., T. Kasumov, A. J. McCullough, N. N. Zein, and J. P. Kirwan. 2012. Role of ceramides in nonalcoholic fatty liver disease. Trends Endocrinol. Metab. 23:365-371.

Phipps, Z. C., F. Seck, A. N. Davis, J. E. Rico, and J. W. McFadden. 2017. Characterization of ceramide in bovine lipoproteins. J. Dairy Sci. 100:8602-8608.

Powell, D. J., E. Hajduch, G. Kular, and H. S. Hundal. 2003. Ceramide disables 3-phosphoinositide binding to the pleckstrin homology domain of protein kinase B (PKB)/Akt by a $\mathrm{PKC} \zeta$-dependent mechanism. Mol. Cell. Biol. 23:7794-7808.

Powell, D. J., S. Turban, G. Alexander, E. Hajduch, and H. S. Hundal. 2004. Intracellular ceramide synthesis and protein kinase $\mathrm{C} \zeta$ activation play an essential role in palmitate-induced insulin resistance in rat L6 skeletal muscle cells. Biochem. J. 382:619-629.

Ren, M. Q., J. Wegner, O. Bellmann, G. A. Brockmann, F. Schneider, F. Teuscher, and K. Ender. 2002. Comparing mRNA levels of genes encoding leptin, leptin receptor, and lipoprotein lipase between dairy and beef cattle. Domest. Anim. Endocrinol. 23:371-381.

Rico, J. E., V. V. R. Bandaru, J. M. Dorskind, N. J. Haughey, and J. W. McFadden. 2015. Plasma ceramides are elevated in overweight Holstein dairy cows experiencing greater lipolysis and insulin resistance during the transition from late pregnancy to early lactation. J. Dairy Sci. 98:7757-7770.

Rico, J. E., A. T. Mathews, J. Lovett, N. J. Haughey, and J. W. McFadden. 2016. Palmitic acid feeding increases ceramide supply in association with increased milk yield, circulating nonesterified fatty acids, and adipose tissue responsiveness to a glucose challenge. J. Dairy Sci. 99:8817-8830.

Rico, J. E., D. E. Rico, Z. C. Phipps, Q. Zeng, B. A. Corl, P. Y. Chouinard, R. Gervais, and J. W. McFadden. 2017a. Circulating ceramide concentrations are influenced by saturated fatty acid chain length in mid-lactation dairy cows. J. Dairy Sci. 100(E-Suppl. 2):394. (Abstr.)

Rico, J. E., S. Saed Samii, A. T. Mathews, J. Lovett, N. J. Haughey, and J. W. McFadden. 2017b. Temporal changes in sphingolipids and systemic insulin sensitivity during the transition from gestation to lactation. PLoS One 12:e0176787.

Schneider, C. A., W. S. Rasband, and K. W. Eliceiri. 2012. NIH Image to Image J: 25 years of image analysis. Nat. Methods 9:671-675.

Schubert, K. M., M. P. Scheid, and V. Duronio. 2000. Ceramide inhibits protein kinase B/Akt by promoting dephosphorylation of serine 473. J. Biol. Chem. 275:13330-13335.

Shah, C., G. Yang, I. Lee, J. Bielawski, Y. A. Hannun, and F. Samad. 2008. Protection from high fat diet-induced increase in ceramide in mice lacking plasminogen activator inhibitor 1. J. Biol. Chem. 283:13538-13548.

Summers, S. A. 2006. Ceramides in insulin resistance and lipotoxicity. Prog. Lipid Res. 45:42-72. 